\title{
Solar neutrino event spectra: Tuning SNO to equalize Super-Kamiokande
}

\author{
G.L. Fogli, E. Lisi, A. Palazzo \\ Dipartimento di Fisica and Sezione INFN di Bari, \\ Via Amendola 173, I-70126 Bari, Italy \\ F.L. Villante \\ Dipartimento di Fisica and Sezione INFN di Ferrara, \\ Via del Paradiso 12, I-44100 Ferrara, Italy
}

\begin{abstract}
The Super-Kamiokande (SK) and the Sudbury Neutrino Observatory (SNO) experiments are monitoring the flux of ${ }^{8} \mathrm{~B}$ solar neutrinos through the electron energy spectrum from the reactions $\nu_{e, \mu, \tau}+e^{-} \rightarrow \nu_{e, \mu, \tau}+e^{-}$and $\nu_{e}+d \rightarrow p+$ $p+e^{-}$, respectively. We show that the $\mathrm{SK}$ detector response to ${ }^{8} \mathrm{~B}$ neutrinos in each bin of the electron energy spectrum (above $8 \mathrm{MeV}$ ) can be approximated, with good accuracy, by the SNO detector response in an appropriate electron energy range (above $5.1 \mathrm{MeV}$ ). For instance, the SK response in the bin $[10,10.5] \mathrm{MeV}$ is reproduced ("equalized") within $\sim 2 \%$ by the SNO response in the range $[7.1,11.75] \mathrm{MeV}$. As a consequence, in the presence of active neutrino oscillations, the SK and SNO event rates in the corresponding energy ranges turn out to be linearly related, for any functional form of the oscillation probability. Such equalization is not spoiled by the possible contribution of hep neutrinos (within current phenomenological limits). In perspective, when the SK and the SNO spectra will both be measured with high accuracy, the SK-SNO equalization can be used to determine the absolute ${ }^{8} \mathrm{~B}$ neutrino flux, and to cross-check the (non)observation of spectral deviations in SK and SNO. At present, as an exercise, we use the equalization to "predict" the SNO energy spectrum, on the basis of the current SK data. Finally, we briefly discuss some modifications or limitations of our results in the case of sterile $\nu$ oscillations and of relatively large Earth matter effects.
\end{abstract}

PACS number(s): 26.65.+t, 13.15.+g, 14.60.Pq 


\section{INTRODUCTION}

The origin of the deficit of solar neutrino events [1 6] with respect to the standard solar model (SSM) predictions [7] 9] is expected to be clarified by the measurement of events induced by ${ }^{8} \mathrm{~B}$ neutrinos in the Super-Kamiokande (SK) [10] and Sudbury Neutrino Observatory $(\mathrm{SNO})[1]$ experiments.

The SK experiment has presented accurate, high-statistics measurements of the energy spectrum of electrons [10] produced by the elastic scattering (ES) reaction

$$
\nu_{e, a}+e^{-} \rightarrow \nu_{e, a}+e^{-}
$$

which can proceed either through $\nu_{e}$ or, in the presence of flavor oscillations, also through the other two active neutrinos $\nu_{a}(a=\mu, \tau)$, with cross sections $\sigma^{e}$ and $\sigma^{a}$, respectively.

The SNO experiment has presented preliminary results of the energy spectrum of electrons [11] produced by the charged-current (CC) absorption reaction

$$
\nu_{e}+d \rightarrow p+p+e^{-}
$$

which can proceed only through $\nu_{e}$, with cross section $\sigma^{c}$.

Both in SK and in SNO, the neutrino interaction process, as well as the subsequent electron detection, tends to degrade the original $\nu$-spectrum information in the final $e$ spectrum, according to the following "energy flow":

$$
E_{\nu} \text { spectrum } \stackrel{\text { int. }}{\longrightarrow} E_{e}^{\prime} \text { spectrum } \stackrel{\text { det. }}{\longrightarrow} E_{e} \text { spectrum }
$$

where $E_{\nu}$ is the $\nu$ energy, $E_{e}^{\prime}$ is the total (true) electron energy, and $E_{e}$ is the total (measured) electron energy. The latter two energies are generally different, as a consequence of the finite detector energy resolution [12 14] associated to the photon counting statistics. As far as one is concerned with the electron event rates in a given energy range $\left[E_{e}^{\min }, E_{e}^{\max }\right]$, the above energy transfer can be completely characterized in terms of the so-called response function $\rho\left(E_{\nu}\right)$ associated to such range and to the ${ }^{8} \mathrm{~B}$ neutrino source [13,15].1

In this work, we analyze the SK and SNO response function to ${ }^{8} \mathrm{~B}$ neutrinos in specific energy ranges. In Sec. II we present our basic result: for each spectrum bin $\left[E_{e}^{\min }, E_{e}^{\max }\right]$ in SK (above $8 \mathrm{MeV}$ ), we find a suitable energy range $\left[\tilde{E}_{e}^{\min }, \tilde{E}_{e}^{\max }\right]$ in SNO (above $\left.5.1 \mathrm{MeV}\right) \mathrm{Z}^{\mathbb{Z}}$ where the corresponding SK and SNO response functions are equal within a few percent. As a consequence of such approximate "equalization", we show in Sec. III that, in the presence of active neutrino oscillations, the SK and SNO event rates in the corresponding energy ranges are linked by a one-to-one (linear) relation, independently of the functional form of the oscillation probability. Moreover, such relation allows the determination of

\footnotetext{
${ }^{1}$ The response function basically represents the (unoscillated) spectrum of interacted neutrinos, in contrast with the unobservable spectrum of emitted solar neutrinos 13, 15.

${ }^{2}$ In the context of this paper, it is useful to adopt a different notation for the observed electron energy in $\operatorname{SK}\left(E_{e}\right)$ and $\operatorname{SNO}\left(\tilde{E}_{e}\right)$.
} 
the absolute ${ }^{8} \mathrm{~B} \nu$ flux. In perspective, the SK-SNO spectral equalization will be useful to cross-check possible (non)observations of spectral deviations in the two experiments, as well as to determine the absolute ${ }^{8} \mathrm{~B} \nu$ flux independently of the neutral-current (NC) SNO measurement (to be performed in the near future [11]). At present, pending both NC data and official CC data from SNO, we use in Sec. III the SK-SNO relation to "predict" the CC spectrum in SNO, on the basis of the current SK energy spectrum. In Sec. IV we show that the previous results are not spoiled by the possible contribution of hep neutrinos (within present phenomenological limits). Finally, we discuss in Sec. V some modifications or limitations of our results in the presence of sterile $\nu$ oscillations and of relatively large Earth matter effects. Conclusions are presented in Sec. VI.

The present work builds upon a previous paper 15] in which a similar strategy was devised to put in one-to-one relation the total event rates of SK and SNO, rather than their spectral rates in each energy bin. The reader is referred to the bibliography in 15 for earlier (but less realistic) attempts to find relations between SK and SNO (spectral) rates in a model-independent way.

\section{SK AND SNO RESPONSE FUNCTIONS}

\section{A. Definitions}

As mentioned in the Introduction, the response function $\rho\left(E_{\nu}\right)$ characterizes the interaction+detection process for a given solar $\nu$ source $\left({ }^{8} \mathrm{~B}\right.$ neutrinos in our case) and for a given electron energy interval $\left(\left[E_{e}^{\min }, E_{e}^{\max }\right]\right.$ in SK and $\left[\tilde{E}_{e}^{\min }, \tilde{E}_{e}^{\max }\right]$ in SNO). The three classes of response functions relevant for our work are

$$
\begin{aligned}
& \rho_{B}^{e}\left(E_{\nu},\left[E_{e}^{\min }, E_{e}^{\max }\right]\right)=\operatorname{SK}\left(\nu_{e}, e\right) \text { ES response function }, \\
& \rho_{B}^{a}\left(E_{\nu},\left[E_{e}^{\min }, E_{e}^{\max }\right]\right)=\operatorname{SK}\left(\nu_{a}, e\right) \text { ES response function }(a=\mu, \tau), \\
& \rho_{B}^{c}\left(E_{\nu},\left[\tilde{E}_{e}^{\min }, \tilde{E}_{e}^{\max }\right]\right)=\operatorname{SNO}\left(\nu_{e}, d\right) \text { CC response function } .
\end{aligned}
$$

Such functions are defined in terms of the standard ${ }^{8} \mathrm{~B}$ energy spectrum $\lambda_{B}\left(E_{\nu}\right)$ [16], of the differential cross sections $\left(d \sigma^{e} / d E_{e}^{\prime}\right.$ and $d \sigma^{a} / d E^{\prime} e$ for elastic scattering 17 and $d \sigma^{c} / d \tilde{E}_{e}^{\prime}$ for CC absorption [18]), and of the detector resolution functions $\left(R_{\mathrm{SK}}[19]\right.$ and $R_{\mathrm{SNO}}[20]$ ):

$$
\begin{aligned}
\rho_{B}^{e}= & \frac{\lambda_{B}\left(E_{\nu}\right) \int_{E_{e}^{\min }}^{E_{e}^{\max }} d E_{e} \int_{0}^{E_{\nu}} d E_{e}^{\prime} \frac{d \sigma^{e}\left(E_{\nu}, E_{e}^{\prime}\right)}{d E_{e}^{\prime}} R_{\mathrm{SK}}\left(E_{e}, E_{e}^{\prime}\right)}{\sigma_{B}^{e}\left[E_{e}^{\min }, E_{e}^{\max ]}\right.}, \\
\rho_{B}^{a}= & \frac{\lambda_{B}\left(E_{\nu}\right) \int_{E_{e}^{\min }}^{E_{e}^{\max }} d E_{e} \int_{0}^{E_{\nu}} d E_{e}^{\prime} \frac{d \sigma^{a}\left(E_{\nu}, E_{e}^{\prime}\right)}{d E_{e}^{\prime}} R_{\mathrm{SK}}\left(E_{e}, E_{e}^{\prime}\right)}{\sigma_{B}^{a}\left[E_{e}^{\min }, E_{e}^{\max }\right]}, \\
\rho_{B}^{c}= & \frac{\lambda_{B}\left(E_{\nu}\right) \int_{\tilde{E}_{e}^{\min }}^{\tilde{E}_{e}^{\max }} d \tilde{E}_{e} \int_{0}^{E_{\nu}} d \tilde{E}_{e}^{\prime} \frac{d \sigma^{c}\left(\tilde{E}_{\nu}, \tilde{E}_{e}^{\prime}\right)}{d \tilde{E}_{e}^{\prime}} R_{\mathrm{SNO}}\left(\tilde{E}_{e}, \tilde{E}_{e}^{\prime}\right)}{\sigma_{B}^{c}\left[\tilde{E}_{e}^{\min }, \tilde{E}_{e}^{\max }\right]},
\end{aligned}
$$


where the denominators $\sigma_{B}^{e, a, c}$ represent the ${ }^{8} \mathrm{~B}$ neutrino cross sections for producing an electron with observed energy in the specified range, as obtained by integrating over $E_{\nu}$ the corresponding numerators in Eqs. (7)-(9).

Throughout this paper, the response functions are calculated through numerical integration of the above expressions, Eqs. (7)-(9). Alternatively, the response functions can be obtained through MonteCarlo (MC) simulations. In particular, the function $\rho_{B}^{e}$ is just the normalized $E_{\nu}$ spectrum (histogram) of the ${ }^{8} \mathrm{~B}$ neutrinos originating a simulated electron with a measured energy $E_{e} \in\left[E_{e}^{\min }, E_{e}^{\max }\right]$ in the SK detector, in the absence of oscillations; analogously for $\rho_{B}^{c}$ in SNO. The function $\rho_{B}^{a}$ can be obtained in the same way as $\rho_{B}^{e}$, provided that the differential cross section $d \sigma^{e} / d E_{e}^{\prime}$ is replaced by $d \sigma^{a} / d E_{e}^{\prime}$ in the MC simulation. Eventually, if our results were adopted by the SK and SNO collaborations in a joint SK-SNO analysis, the corresponding energy ranges and response functions should be optimally calculated and compared through MC simulations, so as to include also those minor detector features (small energy variations of efficiencies, slightly nongaussian resolutions, etc.) which require the knowledge of experimental details beyond the scope of this paper.

\section{B. Correspondence between SK and SNO energy ranges}

In 15] it has been shown that the SK and SNO response functions for the whole spectrum can be equalized, to a good approximation, by an appropriate choice of the corresponding electron energy thresholds. In this work we make an important further step, by showing that the SK response function in each bin $\left[E_{e}^{\min }, E_{e}^{\max }\right]$ (above $8 \mathrm{MeV}$ ) is approximately equalized by the SNO response function in a suitably chosen range $\left[\tilde{E}_{e}^{\min }, \tilde{E}_{e}^{\max }\right]$,

$$
\rho_{B}^{e, a}\left(E_{\nu},\left[E_{e}^{\min }, E_{e}^{\max }\right]\right) \simeq \rho_{B}^{c}\left(E_{\nu},\left[\tilde{E}_{e}^{\min }, \tilde{E}_{e}^{\max }\right]\right) .
$$

In this way one can extend the SK-SNO correspondence from total rates 15 to energy spectra.

Technically, for each SK bin $\left[E_{e}^{\min }, E_{e}^{\max }\right]$, we have determined the extrema of the SNO range $\left[\tilde{E}_{e}^{\min }, \tilde{E}_{e}^{\max }\right]$ by requiring minimization of the integral difference

$$
\Delta=\int d E_{\nu}\left|\rho_{B}^{e}\left(E_{\nu}\right)-\rho_{B}^{c}\left(E_{\nu}\right)\right|
$$

which would be zero if Eq. (10) were exactly satisfied. The results are given in Table I and in Figs. 1 and 2, as we now discuss.

Table I gives, for each SK spectrum bin above $8 \mathrm{MeV}$ (labelled by a sequential number $i=1,2, \ldots, 13)$, the corresponding energy range $\left[\tilde{E}_{e}^{\min }, \tilde{E}_{e}^{\max }\right]$ in SNO (third column) where the SK-SNO response function difference $\Delta$ (fourth column) is minimized. In addition, the fifth and sixth column present the ${ }^{8} \mathrm{~B}$ neutrino cross sections $\sigma_{B}^{e}$ and $\sigma_{B}^{a}(a=\mu, \tau)$ for electron production in the SK range $\left[E_{e}^{\min }, E_{e}^{\max }\right]$ (together with their ratio in the seventh

\footnotetext{
${ }^{3}$ It follows that the functions $\rho_{B}^{e, a, c}\left(E_{\nu}\right)$ in Eqs. (7)-(9) are normalized to unity.
} 
column), while the last column presents the ${ }^{8} \mathrm{~B}$ neutrino cross sections $\sigma_{B}^{c}$ for CC electron production in the corresponding SNO range $\left[\tilde{E}_{e}^{\min }, \tilde{E}_{e}^{\max }\right]$, as defined in Eqs. (7) $-($ (9) and related comments. Figure 1 shows graphically the SK-SNO energy range correspondence expressed by the first three columns of Table I.f

Figure 2 shows the approximate equality between the SK response functions $\rho_{B}^{e, a}$ (solid curves) and the corresponding SNO response functions $\rho_{B}^{c}$ (dotted curves) in a representative subset of energy ranges. In the worst case $(i=13$, highest energy range) the integral difference $\Delta$ does not exceed $10 \%$, and it is often much smaller in other ranges. It can be seen, for instance, that $\rho_{B}^{e}$ for the interval $E_{e} \in[10,10.5] \mathrm{MeV}$ in $\mathrm{SK}(i=5)$ is almost coincident with $\rho_{B}^{c}$ for the interval $\tilde{E}_{e} \in[7.1,11.75], \Delta$ being as small as $2.1 \%$ (see Table I). $]$ In addition, the functions $\rho_{B}^{e}$ and $\rho_{B}^{a}$ in Fig. 1 are graphically indistinguishable (they practically coincide with one and the same solid curve for any range).

We can summarize such results by stating that there is a set $(i=1,2, \ldots, 13)$ of SK and SNO ranges $\left(\left[E_{e}^{\min }, E_{e}^{\max }\right]\right.$ and $\left[\tilde{E}_{e}^{\min }, \tilde{E}_{e}^{\max }\right]$, respectively) where the two experiments are characterized by very similar responses to ${ }^{8} \mathrm{~B}$ neutrinos,

$$
\begin{aligned}
\rho_{B}^{e}\left(E_{\nu},\left[E_{e}^{\min }, E_{e}^{\max }\right]_{i}\right) & =\rho_{B}^{a}\left(E_{\nu},\left[E_{e}^{\min }, E_{e}^{\max }\right]_{i}\right), \\
\rho_{B}^{e}\left(E_{\nu},\left[E_{e}^{\min }, E_{e}^{\max }\right]_{i}\right) & \simeq \rho_{B}^{c}\left(E_{\nu},\left[\tilde{E}_{e}^{\min }, \tilde{E}_{e}^{\max }\right]_{i}\right) .
\end{aligned}
$$

A few comments are in order. The response function shape is governed by the electron energy window $\left[E_{e}^{\min }, E_{e}^{\max }\right]$ and by three functions: the $\nu$ source spectrum $\lambda_{B}$, the normalized differential cross section $\sigma_{B}^{-1} d \sigma_{B} / d E_{e}^{\prime}$, and the detector resolution $R\left(E_{e}^{\prime}, E_{e}\right)$ [see Eqs. (7)-(9)]. Both in SK and in SNO, the bell-shaped functions $\lambda_{B}$ and $R$ render the response functions also bell-shaped, independently of the functional form of the differential cross section. Such functional form, together with the chosen electron energy window, affects instead the "width at half maximum" of the response function. In SK, the normalized cross sections as a function of the electron energy, $\left(\sigma_{B}^{e}\right)^{-1} d \sigma_{B}^{e} / d E_{e}^{\prime}$ and $\left(\sigma_{B}^{a}\right)^{-1} d \sigma_{B}^{a} / d E_{e}^{\prime}$, are both very similar for any fixed neutrino energy $E_{\nu}$ [17]. Therefore, it is not surprising that Eq. (12) holds with very high accuracy.

Conversely, the approximate equality (13) is nontrivial. In fact, the shape of the normalized CC cross section in SNO is very different from the ES case, being peaked at a total electron energy only slight smaller (by $\sim 1.5 \mathrm{MeV}$ ) than the $\nu$ energy, with a long tail at low $\tilde{E}_{e}^{\prime}[18 \|$. Therefore, if identical electron energy ranges were taken for SK and SNO, $\left[E_{e}^{\min }, E_{e}^{\max }\right]=\left[\tilde{E}_{e}^{\min }, \tilde{E}_{e}^{\max }\right]$, the SNO response function would be much narrower (and peaked to slightly higher neutrino energies) than the SK response function (e.g., compare

\footnotetext{
${ }^{4}$ The SK energy bins below $8 \mathrm{MeV}$ are not considered in Fig. 1 and in Table I, since the corresponding SNO energy ranges would be (partly) below the expected SNO threshold [1] of $\sim 5$ $\mathrm{MeV}$.

${ }^{5}$ Notice that the values of $\Delta_{i}$ in Table I are calculated with the input ingredients described in Sec. II.A. Should such ingredients (e.g., the SNO resolution function) change, the extrema of the SNO ranges $\left[\tilde{E}^{\text {min }}, \tilde{E}^{\text {max }}\right]_{i}$ minimizing $\Delta_{i}$ (and the values of $\Delta_{i}$ themselves) should be recalculated for any $i$-th interval.
} 
Figs. 2 and 7 in [13]). The functional difference in the cross sections and in their $Q$-values (and also, to same extent, the difference in the detectors resolution, $R_{\mathrm{SK}} \neq R_{\mathrm{SNO}}$ ), render the SNO response functions tipically different from those of SK. In order to achieve an $a p$ proximate equalization, as the one shown in Fig. 2 , one needs $\left[E_{e}^{\min }, E_{e}^{\max }\right] \neq\left[\tilde{E}_{e}^{\min }, \tilde{E}_{e}^{\max }\right]$ and, in particular: (i) The SNO range width $\tilde{E}_{e}^{\max }-\tilde{E}_{e}^{\min }$ must be larger than the SK range width $E_{e}^{\max }-E_{e}^{\min }$ (so as to "broaden" the SNO response function); and (ii) The central value of the SNO range must be slightly lower than for SK (so as to tune the SNO response function peak at the same energy as in SK). Indeed, both conditions $(i)$ and $(i i)$ are fulfilled by the correlated SK-SNO energy ranges reported in Table I. We will discuss in the next section how to exploit the approximate equalization of the SK and SNO response functions in such correlated energy ranges.

\section{LINKING SK AND SNO SPECTRAL RATES FOR ACTIVE $\nu$ OSCILLATIONS}

In this section we derive a linear relation between the SK and SNO event rates, valid for any of the thirteen couples of SK-SNO energy ranges reported in Table I, and for any functional form of the active neutrino oscillation probability. We start with the definition of the unoscillated rates, to which the experimental rates are usually normalized.

The standard ${ }^{8} \mathrm{~B}$ neutrino event rate (per target electron, in each $i$-th bin of the SK electron spectrum), in the absence of oscillations, is given by

$$
R_{\mathrm{SK}}^{0, i}=\Phi_{B}^{0} \sigma_{B}^{e, i}
$$

where the cross section $\sigma_{B}^{e, i}$ is given in the 5 th column of Table I, while $\Phi_{B}^{0}$ is the ${ }^{8} \mathrm{~B}$ neutrino flux from the reference standard solar model "BP 2000" 8 , $\Phi_{B}^{0}=5.15 \times 10^{6} \mathrm{~cm}^{-2} \mathrm{~s}^{-1}$. Analogously, the standard rate in the corresponding $i$-th energy range of the SNO spectrum is given by

$$
R_{\mathrm{SNO}}^{0, i}=\Phi_{B}^{0} \sigma_{B}^{c, i}
$$

where our evaluation of the cross section $\sigma_{B}^{c, i}$ is given in the last column of Table I.

In general, the event rates measured in SK and SNO can be different from the previous expectations, as a result of either neutrino oscillations or of deviations from the standard model predictions (or both). Here we assume active neutrino oscillations, described by a generic $\nu_{e}$ survival probability function $P_{e e}\left(E_{\nu}\right) \leq 1$. Deviations from the SSM flux are parametrized through an unknown factor $f_{B}$ multiplying the standard flux $\Phi_{B}^{0}$. The event rates measured in SK and SNO should then be equal to

$$
\begin{aligned}
R_{\mathrm{SK}}^{i} & =R_{\mathrm{SK}}^{e, i}+R_{\mathrm{SK}}^{a, i} \\
& =f_{B} \Phi_{B}^{0}\left[\sigma_{B}^{e, i}\left\langle P_{e e}\right\rangle_{B}^{e, i}+\sigma_{B}^{a, i}\left(1-\left\langle P_{e e}\right\rangle_{B}^{a, i}\right)\right],
\end{aligned}
$$

\footnotetext{
${ }^{6}$ The broadening of the SNO energy ranges with respect to SK implies that the intervals $\left[\tilde{E}^{\mathrm{min}}, \tilde{E}^{\mathrm{max}}\right]_{i}$ are partly overlapping, as also evident from Fig. 1.
} 
and

$$
R_{\mathrm{SNO}}^{i}=f_{B} \Phi_{B}^{0} \sigma_{B}^{c, i}\left\langle P_{e e}\right\rangle_{B}^{c, i},
$$

respectively. The terms $\left\langle P_{e e}\right\rangle_{B}^{X, i}(X=e, a, c)$ represent the average survival probability in the $i$-th bin, weighted by the appropriate ${ }^{8} \mathrm{~B}$ response function,

$$
\left\langle P_{e e}\right\rangle_{B}^{X, i}=\int d E_{\nu} \rho_{B}^{X, i}\left(E_{\nu}\right) P_{e e}\left(E_{\nu}\right) \quad(X=e, a, c) .
$$

Notice that Eqs (17)-(19) are exact, i.e., they are definitions which hold without any approximation.

We now apply the approximate equality of the SK and SNO ${ }^{8} \mathrm{~B}$ response functions expressed by Eqs. (12,13). Such equality implies that the average probabilities in (19) are also approximately equal to each other,

$$
\left\langle P_{e e}\right\rangle_{B}^{i} \equiv\left\langle P_{e e}\right\rangle_{B}^{e, i}=\left\langle P_{e e}\right\rangle_{B}^{a, i} \simeq\left\langle P_{e e}\right\rangle_{B}^{c, i}(i=1, \ldots, 13)
$$

within errors smaller than the corresponding values of $\Delta$ [Eq. (11)] given in Table I:

$$
\delta\left\langle P_{e e}\right\rangle_{B}^{i} \equiv\left|\int d E_{\nu} P_{e e}\left(E_{\nu}\right)\left[\rho^{e, i}\left(E_{\nu}\right)_{B}-\rho_{B}^{c, i}\left(E_{\nu}\right)\right]\right| \leq \int d E_{\nu} P_{e e}\left|\rho_{B}^{e, i}-\rho_{B}^{c, i}\right| \leq \Delta_{i} .
$$

It turns out that the error $\delta\left\langle P_{e e}\right\rangle_{B}^{i}$ is often much smaller than its upper limit $\Delta_{i}$ for typical oscillation solutions of the solar neutrino problem, being usually of $O(1 \%)$.

In other words, Eqs. (20,21) show that, for any $i$-th couple of SK and SNO energy intervals in Table I, the two experiments probe (within a typical accuracy of a percent) one and the same average survival probability $\left\langle P_{e e}\right\rangle_{B}^{i}$ for ${ }^{8} \mathrm{~B}$ neutrinos. Therefore, the normalized SK and SNO $i$-th event rates can be written as

$$
\begin{aligned}
r_{\mathrm{SK}}^{i} & \equiv \frac{R_{\mathrm{SK}}^{i}}{R_{\mathrm{SK}}^{0, i}}=f_{B}\left[\left\langle P_{e e}\right\rangle_{B}^{i}+\frac{\sigma_{B}^{a, i}}{\sigma_{B}^{e, i}}\left(1-\left\langle P_{e e}\right\rangle_{B}^{i}\right)\right], \\
r_{\mathrm{SNO}}^{i} & \equiv \frac{R_{\mathrm{SNO}}^{i}}{R_{\mathrm{SNO}}^{0, i}}=f_{B}\left\langle P_{e e}\right\rangle_{B}^{i} .
\end{aligned}
$$

An immediate consequence is that the presence of NC events in the $i$-th SK bin, due to $\nu_{a}$ interactions $(a=\mu, \tau)$, can emerge in a model-independent way by observing $r_{\mathrm{SK}}^{i}-r_{\mathrm{SNO}}^{i}>0$ :

$$
r_{\mathrm{SK}}^{i}-r_{\mathrm{SNO}}^{i}>0 \Longrightarrow \nu_{e} \rightarrow \nu_{\mu, \tau} \text { channel open . }
$$

By eliminating $\left\langle P_{e e}\right\rangle_{B}^{i}$ from Eqs. (22,23), a linear relation between the SK and SNO normalized rates emerges in each $i$-th couple of energy intervals $(i=1, \ldots, 13)$ :

\footnotetext{
${ }^{7}$ In Eq. (17), the contributions from $\nu_{e}$ and $\nu_{a}(a=\mu, \tau)$ are explicitly separated.

${ }^{8}$ The error $\delta\left\langle P_{e e}\right\rangle$ is smaller than $\Delta_{i}$ even when $P_{e e}$ is largest $\left[P_{e e}\left(E_{\nu}\right) \equiv 1\right.$, no oscillations $]$ since the difference $\rho^{e, i}\left(E_{\nu}\right)_{B}-\rho_{B}^{c, i}\left(E_{\nu}\right)$ changes sign as a function of $E_{\nu}$ (Fig. 2). See also the related discussion in Sec. V of [15].
} 


$$
r_{\mathrm{SK}}^{i}=r_{\mathrm{SNO}}^{i}\left(1-\frac{\sigma_{B}^{a, i}}{\sigma_{B}^{e, i}}\right)+f_{B} \frac{\sigma_{B}^{a, i}}{\sigma_{B}^{e, i}}
$$

where the numerical values of $\sigma_{B}^{a, i} / \sigma_{B}^{e, i}$ are given in the 7 th column of Table I. Notice that, since the above equation does not depend on the average probability $\left\langle P_{e e}\right\rangle_{B}^{i}$, it holds for any functional form of the unaveraged survival probability $P_{e e}\left(E_{\nu}\right)$, i.e., for any active $\nu$ oscillation solution of the solar neutrino problem.

For the benefit of the reader, we have explicited Eq. (25) for each energy range in Table II. In all the equations listed in last column of such Table, the numerical coefficients are obviously very similar, since the ratio $\sigma_{B}^{a, i} / \sigma_{B}^{e, i}$ depends weakly on energy. The nonobvious fact is that such equations hold with good accuracy for any functional form of $P_{e e}\left(E_{\nu}\right)$, provided that the SNO energy ranges are chosen according the third column of Table II, for any fixed SK energy bin in the second column of the same table.

Equation (25), explicited in Table II, represents the main result of our work. It implies a one-to-one correspondence between thirteen couples of SK and SNO electron event rates induced by ${ }^{8} \mathrm{~B}$ neutrinos, independently of the specific active $\nu$ oscillation solution. It also explicitly incorporates possible deviations from the standard ${ }^{8} \mathrm{~B}$ flux through the factor $f_{B}$. The physical content of such result can be expressed as follows: for any $i$-th SK bin above 8 $\mathrm{MeV}$, it turns out that there is a SNO energy interval where the average suppression $\left(\left\langle P_{e e}\right\rangle_{B}^{i}\right)$ of the $\nu_{e}$ flux due to oscillations is the same in the two experiments. Then, modulo factors as $f_{B}$ and $\sigma_{B}^{a, i} / \sigma_{B}^{e, i}$, one can get also the average suppression of the $\nu_{a}$ flux in SK $(a=\mu, \tau)$, and thus a link between the SK and SNO rates which is independent of the specific probability function $P_{e e}\left(E_{\nu}\right)$. We discuss now some possible applications of Eq. (25).

When official CC spectrum data (and thus the $r_{\mathrm{SNO}}^{i}$ 's) will be released by SNO, Eq. (25) will provide a determination of $f_{B}$, namely, of the absolute ${ }^{8} \mathrm{~B}$ flux at the $\operatorname{Sun}\left(f_{B} \times \Phi_{B}^{0}\right)$. This fact was derived in 15] by using total SK and SNO rates with appropriate thresholds; our Table II generalizes such result to several energy spectrum ranges $(i=1, \ldots, 13)$, so that the value of $f_{B}$ will be overconstrained. Notice that such constraints on $f_{B}$ will precede the independent determination of $f_{B}$ through the neutral current (NC) measurement planned in the near future in SNO [11.

Table II can also be used to cross-check possible spectral deviations (or their absence) in SK and SNO. If there is (not) a specific spectral distortion pattern $\left\{r_{\mathrm{SK}}^{i} \neq \text { const }\right\}_{1<i<13}$ in the SK spectrum above $8 \mathrm{MeV}$, a similar pattern must show up, independently of $P_{e e}$, in the sequence $\left\{r_{\mathrm{SNO}}^{i}\right\}_{1 \leq i \leq 13}$ above $5.1 \mathrm{MeV}$ in SNO (within uncertainties), according to Table II. Although it is intuitively clear that, if SK finds hints of spectral deviations, they should also be found by SNO [21], Table II provides a quantitative and well-defined way to make such cross-check, with the great advantage that no prior assumption is needed about the (unknown and unobservable) function $P_{e e}\left(E_{\nu}\right)$.

Pending official and definite $\mathrm{CC}$ spectrum data from SNO f we use Table II to make a sort of "prediction" for the SNO spectrum, based on the measured SK spectrum [22]. Figure 3 shows, in the upper panel, the normalized SK spectrum $r_{\text {SK }}^{i}$ [22] above $8 \mathrm{MeV}$.

\footnotetext{
${ }^{9}$ Preliminary SNO results have been reported in [11].
} 
In each of the thirteen bins $\left[E^{\min }, E^{\max }\right]_{i}$, the height of the grey box is equal to the $\pm 1 \sigma$ statistical error $\delta r_{\mathrm{SK}}^{i}$. Inverting the relations in Table II, and assuming $f_{B}=1$, we propagate the SK data set $\left\{r_{\mathrm{SK}}^{i} \pm \delta r_{\mathrm{SK}}^{i}\right\}_{i}$ to a set of "predicted rates" $\left\{r_{\mathrm{SNO}}^{i} \pm \delta r_{\mathrm{SNO}}^{i}\right\}_{i}$ for SNO, in the corresponding energy intervals $\left[\tilde{E}^{\mathrm{min}}, \tilde{E}^{\mathrm{max}}\right]_{i}$. The results are shown in the lower panel of Fig. 3, where the grey boxes have now a width determined by $\left[\tilde{E}^{\text {min }}, \tilde{E}^{\text {max }}\right]_{i}$, and are thus partially overlapping. The meaning of such exercise is the following: In the presence of generic active $\nu$ oscillations, and for $f_{B}=1$, the measured SNO spectrum has to be consistent with the lower panel of Fig. 3, in order to be compatible with the present SK data and their $\pm 1 \sigma$ (stat.) errors. The exercise in Fig. 3 can be repeated for $f_{B} \neq 1$ (not shown).

A final remark is in order, concerning the uncertainties affecting Eq. (25). Besides the experimental uncertainties in the SK and SNO rates, the main theoretical uncertainty is related to the absolute normalization of the $\sigma^{c}$ cross section for CC interactions in SNO, which enters in the calculation of $R_{\text {SNO }}^{0, i}$ [Eq. (15)] and thus propagates to the normalized rate $r_{\mathrm{SNO}}^{i}[\mathrm{Eq} .(23)]$. Such error is estimated in [23 to be $\sim 6 \%$ at $1 \sigma$, by comparing different calculations; a more definite evaluation (and possibly a reduction) would be highly desirable. Compared with such uncertainty, the error induced by the approximate SK-SNO equalization [Eq. (21)] is typically less relevant, except perhaps in the worst case $(i=13$ energy range). Finally, notice that Table II involves thirteen couples of quantities, which have correlated errors. Besides the obvious bin-by-bin correlation of detector systematics in SK (and, independently, in SNO), the ${ }^{8} \mathrm{~B}$ spectrum shape uncertainty [16] represents a systematic error in common to SK and SNO. Moreover, since the energy ranges $\left[\tilde{E}^{\text {min }}, \tilde{E}^{\text {max }}\right]_{i}$ partially overlap in SNO (Fig. 1), the corresponding SNO rates $r_{\mathrm{SNO}}^{i}$ are also statistically correlated. All such "complications" of a joint SK-SNO analysis can actually be handled by standard statistical techniques (e.g., covariance matrices); however, pending detailed data and official evaluations of uncertainties in SNO, we do not furtherly explore this matter at present.

\section{EFFECT OF hep NEUTRINOS}

In this section we show that our main result [Eq. (25)] is basically preserved in the presence of a nonnegligible hep neutrino contribution to the SK and SNO event rates, modulo a redefinition of the factor $f_{B}$.

The latest evaluation (BP 2000) of the standard hep flux is $\Phi_{h e p}^{0}=9.3 \times 10^{-7} \mathrm{~cm}^{-2} \mathrm{~s}^{-1}$ [8], with large (unquoted) uncertainties. We parametrize such uncertainty by introducing (analogously to $f_{B}$ ) a free parameter $f_{\text {hep }}$ multiplying $\Phi_{h e p}^{0}$. The neutrino energy spectrum at the Sun (as far as SK and SNO are concerned) becomes then:

$$
\Phi\left(E_{\nu}\right)=f_{B} \Phi_{B}^{0} \lambda_{B}\left(E_{\nu}\right)+f_{h e p} \Phi_{h e p}^{0} \lambda_{h e p}\left(E_{\nu}\right),
$$

where $\lambda_{h e p}\left(E_{\nu}\right)$ is the hep neutrino energy spectrum [9].

We recall that the slight "excess" of events in the high-energy tail of the SK normalized spectrum [10] is roughly consistent with the standard contribution $\left(f_{\text {hep }} \sim 1\right)$ from hep neutrinos [8], and provides a 90\% C.L. upper bound to such contribution,

$$
f_{\text {hep }} \lesssim 3
$$


as derived in [10] through an analysis with unconstrained $f_{B}$. As an example of relatively "large" hep flux we consider then the reference case $\left(f_{B}, f_{\text {hep }}\right)=(1,3)$.

As for ${ }^{8} \mathrm{~B}$ neutrinos [Eqs. (4)-(河)], one can introduce for hep neutrinos three new response functions,

$$
\rho_{\text {hep }}^{X}\left(E_{\nu}\right)=\text { response function to hep neutrinos }(X=e, a, c) \text {, }
$$

which are defined analogously to Eqs. (7)-(9), modulo the replacement of $\lambda_{B}\left(E_{\nu}\right)$ with $\lambda_{h e p}\left(E_{\nu}\right)$. Correspondingly, one can define three new averaged probabilities,

$$
\left\langle P_{e e}\right\rangle_{h e p}^{X}=\int d E_{\nu} \rho_{h e p}^{X}\left(E_{\nu}\right) P_{e e}\left(E_{\nu}\right) \quad(X=e, a, c)
$$

Figure 4 shows the SK response functions $\rho_{h e p}^{e}$ and $\rho_{\text {hep }}^{a}$ (graphically coincident with the solid curve) in three representative high-energy bins, together with the SNO response function $\rho_{\text {hep }}^{c}$ (dotted curve) in the corresponding energy ranges given in Table I. The response functions are somewhat different from each other, the difference becoming rapidly larger in lower energy intervals (not shown). Therefore, we cannot derive a reasonably approximate equality of the kind $\left\langle P_{e e}\right\rangle_{h e p}^{e} \simeq\left\langle P_{e e}\right\rangle_{h e p}^{c}$ [as it was instead the case for ${ }^{8} \mathrm{~B}$ neutrinos, Eq.(20)] However, this potential problem practically disappears in the combination with ${ }^{8} \mathrm{~B}$ neutrinos.

In fact, let us consider the combined $\mathrm{B}+$ hep response functions $\rho^{X}$ for a source flux as in Eq. (26). Such functions are given by

$$
\begin{aligned}
\rho^{X}\left(E_{\nu}\right)= & (\mathrm{B}+h e p) \text { response function }(X=e, a, c) \\
= & \frac{f_{B} \Phi_{B}^{0} \sigma_{B}^{X} \rho_{B}^{X}\left(E_{\nu}\right)+f_{h e p} \Phi_{h e p}^{0} \sigma_{h e p}^{X} \rho_{h e p}^{X}\left(E_{\nu}\right)}{f_{B} \Phi_{B}^{0} \sigma_{B}^{X}+f_{h e p} \Phi_{h e p}^{0} \sigma_{h e p}^{X}} \\
= & \frac{\rho_{B}^{X}\left(E_{\nu}\right)+\frac{f_{h e p} \Phi_{h e p}^{0} \sigma_{h e p}^{X}}{f_{B} \Phi_{B}^{0} \sigma_{B}^{X}} \rho_{h e p}^{X}\left(E_{\nu}\right)}{1+\frac{f_{h e p} \Phi_{h e p}^{0} \sigma_{h e p}^{X}}{f_{B} \Phi_{B}^{0} \sigma_{B}^{X}}},
\end{aligned}
$$

where our evaluations of the cross sections $\sigma_{\text {hep }}^{X}$ (defined in a way analogous to $\sigma_{B}^{X}$ ) are given in the second, third, and fourth column of Table III for $X=e, a, c$, respectively. In the same table, we also report the ratios between the standard contributions of hep neutrinos $\left(\Phi_{\text {hep }}^{0} \sigma_{\text {hep }}^{X}\right)$ to ${ }^{8} \mathrm{~B}$ neutrinos $\left(\Phi_{B}^{0} \sigma_{B}^{X}\right)$ in each $i$-th energy range. Since such ratios are small, the combined response functions $\rho^{X}$ [Eq. (31)] are always dominated by the $\rho_{B}^{X}$ component, even for a relatively large $h e p$ flux (e.g., $f_{h e p} / f_{B}=3$ ). Therefore, we expect that, although the SK and SNO response functions to hep neutrinos can be noticeably different (see Fig. 4), the combined response functions to $(\mathrm{B}+$ hep) neutrinos can still be taken as approximately equal in SK-SNO corresponding ranges. Such expectation is confirmed by Fig. 5, were the combined response functions are shown in the same representative energy ranges of Fig. 2, but for the reference case of relatively large hep flux $\left(f_{B}, f_{\text {hep }}\right)=(1,3)$. The SK

\footnotetext{
${ }^{10}$ One can appreciate the contribution of hep neutrinos in the upper tail of the response functions by comparing Fig. $5\left(f_{\text {hep }}=3\right)$ with Fig. $2\left(f_{\text {hep }}=0\right)$.
} 
and SNO combined response functions (solid and dotted curves, respectively) are indeed approximately equal in any energy range, the integral difference $\Delta$ being a few percent (as reported in the last column of Table III), except for the last bin where it exceeds $10 \%$. We remind that the situation would be better (smaller $\Delta$ ) for the phenomenologically preferred case [10] of $f_{h e p} / f_{B}<3$. Therefore, if we average the oscillation probability over the combined response functions in each $i$-th energy range,

$$
\left\langle P_{e e}\right\rangle^{X, i}=\int d E_{\nu} \rho^{X, i}\left(E_{\nu}\right) P_{e e}\left(E_{\nu}\right)
$$

we can still assume them as approximately equal to each other,

$$
\left\langle P_{e e}\right\rangle^{i} \equiv\left\langle P_{e e}\right\rangle^{e, i}=\left\langle P_{e e}\right\rangle^{a, i} \simeq\left\langle P_{e e}\right\rangle^{c, i}(i=1, \ldots, 13)
$$

as it was the case for ${ }^{8} \mathrm{~B}$ neutrinos only [Eq. (20)]. Finally, we remark that the approximate equalization of the combined SK and SNO response functions in Fig. 5 is partly due also to the fact that the relative $h e p$ contribution in each energy range is approximately independent of the interaction process $X(=e, a, c)$,

$$
\epsilon_{i} \equiv \frac{\Phi_{h e p}^{0} \sigma_{h e p}^{e}}{\Phi_{B}^{0} \sigma_{B}^{e}}=\frac{\Phi_{h e p}^{0} \sigma_{h e p}^{a}}{\Phi_{B}^{0} \sigma_{B}^{a}} \simeq \frac{\Phi_{h e p}^{0} \sigma_{h e p}^{c}}{\Phi_{B}^{0} \sigma_{B}^{c}}
$$

the differences being a few $\times 10^{-3}$ (compare the 5th, 6th, and 7th columns of Table III), except, once again, for the last range $(i=13)$, where the above approximation is not as good.

Let us discuss the effect of a nonzero hep flux on the results of the previous section. We redefine the expected normalized rates $r_{\mathrm{SK}}^{i}$ and $r_{\mathrm{SNO}}^{i}$ as in Eqs. (22,23), with denominators $R_{\mathrm{SK}}^{0, i}$ and $R_{\mathrm{SNO}}^{0, i}$ still given by the standard rate from ${ }^{8} \mathrm{~B}$ neutrinos only. Eqs. (14) and (15). However, we now include the hep neutrino contribution in the numerators $R_{\mathrm{SK}}^{i}$ and $R_{\mathrm{SNO}}^{i}$ :

$$
\begin{aligned}
R_{\mathrm{SK}}^{i} & =f_{B} \Phi_{B}^{0}\left[\sigma_{B}^{e, i}\left\langle P_{e e}\right\rangle_{B}^{e, i}+\sigma_{B}^{a, i}\left(1-\left\langle P_{e e}\right\rangle_{B}^{a, i}\right)\right]+(B \leftrightarrow h e p), \\
R_{\mathrm{SNO}}^{i} & =f_{B} \Phi_{B}^{0} \sigma_{B}^{c, i}\left\langle P_{e e}\right\rangle_{B}^{e, i}+(B \leftrightarrow h e p) .
\end{aligned}
$$

The above Eqs. (35, 36) are exact, i.e., they do not imply any approximation. By applying to such equations the approximate equalities expressed by Eqs. (33) and (34) we obtain, after some algebra,

$$
\begin{aligned}
r_{\mathrm{SK}}^{i} & =\left(f_{B}+\epsilon_{i} f_{h e p}\right)\left[\left\langle P_{e e}\right\rangle^{i}+\frac{\sigma_{B}^{a, i}}{\sigma_{B}^{e, i}}\left(1-\left\langle P_{e e}\right\rangle^{i}\right)\right], \\
r_{\mathrm{SNO}}^{i} & =\left(f_{B}+\epsilon_{i} f_{h e p}\right)\left\langle P_{e e}\right\rangle^{i},
\end{aligned}
$$

\footnotetext{
${ }^{11}$ The "standard" rate to which one should normalize the measured rate may or may not include the SSM hep neutrino contribution (in addition to $\mathrm{SSM}^{8} \mathrm{~B}$ neutrinos), the choice being purely conventional. Our choice (hep neutrinos not included in the standard unoscillated rates $R_{\mathrm{SK}}^{0, i}$ and $R_{\mathrm{SNO}}^{0, i}$ ) leads to more compact expressions in the context of our approach.
} 
from which we can eliminate the average probability $\left\langle P_{e e}\right\rangle^{i}$ to get

$$
r_{\mathrm{SK}}^{i}=r_{\mathrm{SNO}}^{i}\left(1-\frac{\sigma_{B}^{a, i}}{\sigma_{B}^{e, i}}\right)+\left(f_{B}+\epsilon_{i} f_{h e p}\right) \frac{\sigma_{B}^{a, i}}{\sigma_{B}^{e, i}} .
$$

The above equation holds with an accuracy comparable to that of Eq. (25), namely, $O(1 \%)$ for typical solutions to the solar neutrino problem. The only difference with Eq. (25) is the replacement $f_{B} \rightarrow f_{B}+\epsilon_{i} f_{\text {hep }}$, where $\epsilon_{i}$ [defined in Eq. (34)] is tabulated in the fifth column of Table III. Therefore, all the considerations in Sec. IV, related to the existence of a linear relation between SK and SNO rates in appropriate energy ranges, hold also in the presence of a nonnegligible hep neutrino contribution, up to the mentioned replacement for $f_{B}$ (barring perhaps the last range $i=13$, where the approximations may not be particularly good).

In addition, suppose that both the $r_{\mathrm{SK}}^{i}$ 's and the $r_{\mathrm{SNO}}^{i}$ 's are measured with high precision, and that $f_{B}$ is also precisely determined from the NC measurement in SNO [11]: then the only unknown would be $f_{\text {hep }}$, which could be hopefully determined by applying Eq. (39) to the highest energy ranges (say, $i=11,12$, and possibly 13) where $\epsilon_{i}$ is largest. Of course, at the level of accuracy required to determine the absolute hep neutrino flux $f_{\text {hep }} \Phi_{\text {hep }}^{0}$ from the SK and SNO data, one needs a very careful estimate of all the uncertainties involved, including the intrinsic approximations of our approach.

\section{EFFECT OF EARTH MATTER AND OF STERILE NEUTRINOS}

In this section we briefly discuss some limitations or modifications of our results, which can arise when neutrino oscillations are affected by Earth matter effects [24] 26], or by transitions to a sterile state $\nu_{s}$.

\section{A. Earth matter effects}

So far, we have implicitly assumed one and the same probability function $P_{e e}\left(E_{\nu}\right)$ for both SK and SNO. This assumption is no longer valid in the presence of sizable Earth matter effects (during nighttime), since the different SK and SNO latitudes imply different nadir angle $(\eta)$ exposures for the two experiments [27]. Although the SK data have already excluded a significant fraction of the parameter space where Earth matter effects are large [10], one cannot exclude that more precise SK (and SNO) data may still reveal some distortion in the nadir distribution (see, e.g., [23,28] and references therein). In such case, the probability function $P_{e e}$ could be significantly different in SK and SNO, especially along the inner trajectories traversing the Earth core, where mantle+core interference effects can occur [29], and where the exposure functions in SK and SNO are significantly different.

It follows that, if sizable Earth matter effects would emerge in SK or SNO nighttime data, our approach could be strictly applied only to daytime events. Notice, however, that the SK and SNO exposure functions, although different in principle, turn out to be approximately similar in the nadir angle range $0.4 \lesssim \eta \lesssim 0.8$ (see Fig. 3 in [27]). Therefore, modulo such approximation, one could still assume one and the same function $P_{e e}\left(E_{\nu}\right)$ in SK and SNO (and thus apply our results) for the fraction of nighttime data with $\eta \in[0.4,0.8]$. 


\section{B. Sterile neutrino oscillations}

Recent global fits to solar neutrino data, including the latest SK results [10], tend to disfavor pure $\nu_{e} \rightarrow \nu_{s}$ oscillations [10,30] as compared to $\nu_{e} \rightarrow \nu_{\mu, \tau}$, although it is perhaps too early to claim rejection of the $\nu_{s}$ scenario [30]. In any case, mixed (active+sterile) solar neutrino oscillations are certainly still allowed [30], and can also be made consistent with the atmospheric neutrino oscillation evidence [31]. In the general case of active+sterile oscillations, Eqs. (22) and (23) are modified as follows:

$$
\begin{aligned}
r_{\mathrm{SK}}^{i} & =f_{B}\left[\left\langle P_{e e}\right\rangle^{i}+\frac{\sigma_{B}^{a, i}}{\sigma_{B}^{e, i}}\left(1-\left\langle P_{e e}\right\rangle^{i}-\left\langle P_{e s}\right\rangle^{i}\right)\right], \\
r_{\mathrm{SNO}}^{i} & =f_{B}\left\langle P_{e e}\right\rangle^{i},
\end{aligned}
$$

where the unitarity relation $1=P_{e e}+P_{e \mu}+P_{e s}$ has been used, $P_{e s}$ being the $\nu_{e} \rightarrow \nu_{s}$ oscillation probability.[2]

Unfortunately, one cannot eliminate both $\left\langle P_{e e}\right\rangle^{i}$ and $\left\langle P_{e s}\right\rangle^{i}$ from the above equations, so as to derive a model-independent relation between the SK and SNO rates analogous to Eq. (25). However, one can eliminate at least $\left\langle P_{e e}\right\rangle^{i}$ to get the relation

$$
\frac{r_{\mathrm{SK}}^{i}-r_{\mathrm{SNO}}^{i}\left(1-\sigma_{B}^{a, i} / \sigma_{B}^{e, i}\right)}{\sigma_{B}^{a, i} / \sigma_{B}^{e, i}}=f_{B}\left(1-\left\langle P_{e s}\right\rangle^{i}\right),
$$

which might still provide a useful test of $\nu_{e} \rightarrow \nu_{s}$ transitions. In fact, the quantity $f_{B}\left(1-\left\langle P_{e s}\right\rangle^{i}\right)$ is independent of energy range index $i$ only if $P_{e s}\left(E_{\nu}\right)=$ const. Therefore, variations of the left-hand-side of Eq. (42) in different energy ranges would provide a signature of $\nu_{e} \rightarrow \nu_{s}$ transitions with energy-dependent probability.

Further links between $r_{\mathrm{SK}}^{i}$ and $r_{\mathrm{SNO}}^{i}$ can only be obtained by making some assumptions about $P_{e s}$. For instance, by assuming pure sterile oscillations $\left(1=P_{e e}+P_{e s}\right)$, one gets from Eqs (40) and (41) that:

$$
\text { pure } \nu_{e} \rightarrow \nu_{s} \text { oscillations } \Longrightarrow r_{\mathrm{SK}}^{i}=r_{\mathrm{SNO}}^{i}
$$

i.e., the normalized rates in the SK and SNO corresponding ranges are expected to be equal (due to the absence of $\nu_{\mu, \tau} \mathrm{NC}$ contributions in $\mathrm{SK}$ ). The above equation is interesting because, independently of the functional form of $P_{e e}\left(E_{\nu}\right)$, it provides a possible way to distinguish pure sterile oscillations [Eq. (43)] from pure active oscillations [Eq. (25)]. Figure 6 shows, for instance, the "predicted" SNO spectrum for pure $\nu_{e} \rightarrow \nu_{s}$ oscillations, as obtained from Eq. (43). A comparison with Fig. 3 ("predicted" SNO spectrum for active oscillations) shows how the normalized SNO rates are expected to be less suppressed for the sterile case, on the basis of the present SK data. Finally, notice that Eq. (43) does not allow to determine $f_{B}$, nor to discriminate pure sterile oscillations from no oscillations. Such loss of predictive power simply reflects the fact that, for increasing amplitude of the $\nu_{e} \rightarrow \nu_{s}$ channel, there is a decreasing NC contribution in SK, so that the SK and SNO event rates tend to be equally suppressed, their combination providing eventually little additional information.

\footnotetext{
${ }^{12}$ Similar relations were discussed in 15 for the total SK and SNO rates.
} 


\section{CONCLUSIONS}

In this work, we have shown that the SK detector response to ${ }^{8} \mathrm{~B}$ solar neutrinos, in each bin of the electron energy spectrum above $8 \mathrm{MeV}$, can be accurately approximated by the SNO detector response in appropriate (different) electron energy ranges. In a sense, it is possible to "tune" the SNO energy "bandwidth" so as to "equalize" the SK response in various spectrum bins. As a consequence, we have derived a set of linear relations among the SK and SNO spectral rates, whose distinguishing feature is the independence on the functional form of the (active) neutrino oscillation probability. Such relations can be used to determine the absolute ${ }^{8} \mathrm{~B}$ neutrino flux from SK and SNO data, and to cross-check the joint (non)observation of spectral deviations in the two experiments. As an exercise, pending official SNO spectrum data, we have inverted such relations to "predict" the SNO energy spectrum, on the basis of the current SK data. We have also discussed the effects and implications of a nonnegligible hep neutrino flux, of relatively large Earth matter effects, and of sterile neutrino transitions. We conclude by stressing that, should our results be adopted by the SK and SNO collaboration to perform a joint SK-SNO spectral analysis, dedicated MonteCarlo simulations would be desirable to assess all the uncertainties and their correlations, as well as to quantify (through a more detailed description of the instrumental responses) the approximations associated with our approach.

\section{ACKNOWLEDGMENTS}

This work was supported in part by INFN (FA51 Project) and in part by the Italian MURST ("Astroparticle Physics" Project). We thank D. Montanino for useful discussions and suggestions. 


\section{REFERENCES}

[1] Homestake Collaboration, B.T. Cleveland, T.J. Daily, R. Davis Jr., J.R. Distel, K. Lande, C. K. Lee, P.S. Wildenhain, and J. Ullman, Astrophys. J. 496, 505 (1998).

[2] Kamiokande Collaboration, Y. Fukuda et al., Phys. Rev. Lett. 77, 1683 (1996).

[3] SAGE Collaboration, J.N. Abdurashitov et al., Phys. Rev. C 60, 055801 (1999).

[4] GALlEX Collaboration, W. Hampel et al., Phys. Lett. B 447, 127 (1999).

[5] GNO Collaboration, M. Altmann et al., Phys. Lett. B 490, 16 (2000).

[6] Super-Kamiokande Collaboration, Y. Fukuda et al., Phys. Rev. Lett. 82, 2430 (1999).

[7] J. N. Bahcall, Neutrino Astrophysics (Cambridge University Press, Cambridge, England, 1989).

[8] J.N. Bahcall, M.H. Pinsonneault, and S. Basu, astro-ph/0010346.

[9] J.N. Bahcall homepage, http://www.sns.ias.edu/ jnb (Neutrino Software and Data).

[10] Y. Suzuki for the Super-Kamiokande Collaboration, in Neutrino 2000, 19th International Conference on Neutrino Physics and Astrophysics (Sudbury, Canada, 2000) Nucl. Phys. B (Proc. Suppl.) 91, 29 (2001).

[11] A.B. McDonald for the SNO Collaboration, in Neutrino 2000 [10], p. 21.

[12] J.N. Bahcall and E. Lisi, Phys. Rev. D 54, 5417 (1996).

[13] B. Faïd, G.L. Fogli, E. Lisi, and D. Montanino, Phys. Rev. D 55, 1353 (1997).

[14] G.L. Fogli, E. Lisi, and D. Montanino, Astropart. Phys. 9, 119 (1998).

[15] F.L. Villante, G. Fiorentini, and E. Lisi, Phys. Rev. D 59, 013006 (1999).

[16] J.N. Bahcall, E. Lisi, D.E. Alburger, L. De Braeckeleer, S.J. Freedman, and J. Napolitano, Phys. Rev. C 54, 411 (1996).

[17] We adopt the $\nu$-e cross section calculations reported in J.N. Bahcall, M. Kamionkowsky, and A. Sirlin, Phys. Rev. D 51, 6146 (1995). See also M. Passera, hep-ph/0011190, for recent refined calculations of $O(\alpha)$ QED corrections to $\nu$-e scattering.

[18] We adopt the $\nu$ - $d$ CC cross section as reported in the computer code DIFFCROSS.FOR [9, [12], and corresponding to the "Kubodera-Nozawa" calculation option. See also S. Nakamura, T. Sato, V. Gudkov, and K. Kubodera, nucl-th/0009012, for recent refined calculations of $\nu$ - $d$ cross sections.

[19] In the present work, the width of the SK energy resolution function is taken to be $\pm 15 \%$ for an electron (kinetic) energy $10 \mathrm{MeV}$, as in [14]. Such value, which is in agreement with the SK calibration results graphically reported in [6], scales with energy as described in 12,13 .

[20] In the present work, the width of the SNO energy resolution function is taken to be $\pm 11 \%$ for an electron (kinetic) energy of $10 \mathrm{MeV}$, as in [12]. Such value is in agreement with the preliminary SNO electron energy calibration results $(\sim 9$ photomultiplier hits/MeV, as reported in [11]). The value previously adopted in [15] (on the basis of the SNO prospective estimates available at that time) was somewhat larger $( \pm 14 \%)$.

[21] See., e.g., the SK-SNO spectral correlation studies in: S.M. Bilenky and C. Giunti, Astropart. Phys. 2, 353 (1994); K. Kwong amd S.P. Rosen, Phys. Rev. D 51, 6159 (1995); G.L. Fogli, E. Lisi, and D. Montanino, Phys. Lett. B 434, 333 (1998); J.N. Bahcall, P.I. Krastev, and A.Yu. Smirnov, Phys. Rev. D 63, 053012 (2001).

[22] We use the SK spectrum data corresponding to 1117 days of detector exposure [10], normalized to the ${ }^{8} \mathrm{~B}$ neutrino flux from the BP 2000 standard solar model [8]. 
[23] J.N. Bahcall, P.I. Krastev, and A.Yu. Smirnov, Phys. Rev. D 62, 093004 (2000).

[24] L. Wolfenstein, Phys. Rev. D 17, 2369 (1978).

[25] S.P. Mikheyev and A.Yu. Smirnov, Yad. Fiz. 42, 1441 (1985) [Sov. J. Nucl. Phys. 42, 913 (1985)]; Nuovo Cimento C 9, 17 (1986).

[26] V. Barger, S. Pakvasa, R.J.N. Phillips and K. Whisnant, Phys. Rev. D 22, 2718 (1980).

[27] E. Lisi and D. Montanino, Phys. Rev. D 56, 1792 (1997).

[28] M.C. Gonzalez-Garcia, C. Peña-Garay, and A.Yu. Smirnov, hep-ph/0012313.

[29] S.T. Petcov, Phys. Lett. B 434, 321 (1998); E.Kh. Akhmedov, Nucl. Phys. B 538, 25 (1999).

[30] C. Giunti, M.C. Gonzalez-Garcia, and C. Peña-Garay, Phys. Rev. D 62, 013005 (2000); M.C. Gonzalez-Garcia and C. Peña-Garay, hep-ph/0011245.

[31] G.L. Fogli, E. Lisi, and A. Marrone, Phys. Rev. D 63, 053008 (2001). 


\section{TABLES}

TABLE I. SNO electron energy ranges (third column) where the SNO response function to ${ }^{8} \mathrm{~B}$ neutrinos equalizes the SK response function in the $i$-th electron energy bin (second column). The integral difference $\Delta$ between the SK and SNO response functions is given in the fourth column. ${ }^{8} \mathrm{~B}$ neutrino cross sections for electron production in each range (including detector resolution effects) are given in the remaining columns. See the text for details.

\begin{tabular}{cccccccc}
\hline \hline $\begin{array}{c}\text { Range } \\
i\end{array}$ & $\begin{array}{c}{\left[E_{e}^{\min }, E_{e}^{\max }\right]} \\
(\mathrm{SK}, \mathrm{MeV})\end{array}$ & $\begin{array}{c}{\left[\tilde{E}_{e}^{\min }, \tilde{E}_{e}^{\max }\right]} \\
(\mathrm{SNO}, \mathrm{MeV})\end{array}$ & $\begin{array}{c}\Delta \\
\times 100\end{array}$ & $\begin{array}{c}\sigma_{B}^{e} \\
\left(10^{-44} \mathrm{~cm}^{2}\right)\end{array}$ & $\begin{array}{c}\sigma_{B}^{a} \\
\left(10^{-45} \mathrm{~cm}^{2}\right)\end{array}$ & $\begin{array}{c}\sigma_{B}^{a} / \sigma_{B}^{e} \\
\left(10^{-42} \mathrm{~cm}^{2}\right)\end{array}$ \\
\hline 1 & {$[8,8.5]$} & {$[5.10,9.90]$} & 6.9 & 0.1415 & 0.2191 & 0.155 & 0.8092 \\
2 & {$[8.5,9]$} & {$[5.60,10.35]$} & 5.2 & 0.1182 & 0.1819 & 0.154 & 0.7740 \\
3 & {$[9,9.5]$} & {$[6.10,10.80]$} & 3.8 & 0.0970 & 0.1485 & 0.154 & 0.7214 \\
4 & {$[9.5,10]$} & {$[6.60,11.30]$} & 2.8 & 0.0781 & 0.1190 & 0.153 & 0.6570 \\
5 & {$[10,10.5]$} & {$[7.10,11.75]$} & 2.1 & 0.0616 & 0.0934 & 0.152 & 0.5790 \\
6 & {$[10.5,11]$} & {$[7.55,12.30]$} & 1.8 & 0.0475 & 0.0718 & 0.151 & 0.5070 \\
7 & {$[11,11.5]$} & {$[8.05,12.85]$} & 1.8 & 0.0357 & 0.0538 & 0.151 & 0.4222 \\
8 & {$[11.5,12]$} & {$[8.50,13.45]$} & 2.0 & 0.0262 & 0.0394 & 0.150 & 0.3481 \\
9 & {$[12,12.5]$} & {$[8.95,14.35]$} & 2.6 & 0.0186 & 0.0280 & 0.150 & 0.2786 \\
10 & {$[12.5,13]$} & {$[9.45,14.95]$} & 3.1 & 0.0129 & 0.0193 & 0.150 & 0.2087 \\
11 & {$[13,13.5]$} & {$[9.90,18.25]$} & 3.8 & 0.0086 & 0.0129 & 0.150 & 0.1554 \\
12 & {$[13.5,14]$} & {$[10.30,20]$} & 4.4 & 0.0056 & 0.0083 & 0.148 & 0.1159 \\
13 & {$[14,20]$} & {$[11.20,20]$} & 8.6 & 0.0081 & 0.0120 & 0.148 & 0.0532 \\
\hline \hline
\end{tabular}

TABLE II. Linear relations linking the normalized SK and SNO spectral rates (last column) in SK-SNO corresponding energy ranges (reported for completeness in the 2nd and 3rd columns). Such relations hold for any functional form on the $\nu_{e}$ survival probability, assuming active $\nu$ oscillations.

\begin{tabular}{cccl}
\hline \hline $\begin{array}{c}\text { Range } \\
i\end{array}$ & {$\left[E_{e}^{\min }, E_{e}^{\max }\right]$} & {$\left[\tilde{E}_{e}^{\min }, \tilde{E}_{e}^{\max }\right]$} & $\begin{array}{l}\text { Relation between normalized } \\
\text { event rates in SK and SNO }\end{array}$ \\
\hline 1 & $(\mathrm{SK}, \mathrm{MeV})$ & {$[5.10,9.90]$} & $r_{\mathrm{SK}}=0.845 \times r_{\mathrm{SNO}}+0.155 \times f_{B}$ \\
2 & {$[8,8.5]$} & {$[5.60,10.35]$} & $r_{\mathrm{SK}}=0.846 \times r_{\mathrm{SNO}}+0.154 \times f_{B}$ \\
3 & {$[8.5,9]$} & {$[6.10,10.80]$} & $r_{\mathrm{SK}}=0.846 \times r_{\mathrm{SNO}}+0.154 \times f_{B}$ \\
4 & {$[9,9.5]$} & {$[6.60,11.30]$} & $r_{\mathrm{SK}}=0.847 \times r_{\mathrm{SNO}}+0.153 \times f_{B}$ \\
5 & {$[9.5,10]$} & {$[7.10,11.75]$} & $r_{\mathrm{SK}}=0.848 \times r_{\mathrm{SNO}}+0.152 \times f_{B}$ \\
6 & {$[10,10.5]$} & {$[7.55,12.30]$} & $r_{\mathrm{SK}}=0.849 \times r_{\mathrm{SNO}}+0.151 \times f_{B}$ \\
7 & {$[10.5,11]$} & {$[8.05,12.85]$} & $r_{\mathrm{SK}}=0.849 \times r_{\mathrm{SNO}}+0.151 \times f_{B}$ \\
8 & {$[11,11.5]$} & {$[8.50,13.45]$} & $r_{\mathrm{SK}}=0.850 \times r_{\mathrm{SNO}}+0.150 \times f_{B}$ \\
9 & {$[11.5,12]$} & {$[8.95,14.35]$} & $r_{\mathrm{SK}}=0.850 \times r_{\mathrm{SNO}}+0.150 \times f_{B}$ \\
10 & {$[12,12.5]$} & {$[9.45,14.95]$} & $r_{\mathrm{SK}}=0.850 \times r_{\mathrm{SNO}}+0.150 \times f_{B}$ \\
11 & {$[12.5,13]$} & {$[9.90,18.25]$} & $r_{\mathrm{SK}}=0.850 \times r_{\mathrm{SNO}}+0.150 \times f_{B}$ \\
12 & {$[13,13.5]$} & {$[10.30,20]$} & $r_{\mathrm{SK}}=0.852 \times r_{\mathrm{SNO}}+0.148 \times f_{B}$ \\
13 & {$[13.5,14]$} & $r_{\mathrm{SK}}=0.852 \times r_{\mathrm{SNO}}+0.148 \times f_{B}$ \\
\hline \hline
\end{tabular}


TABLE III. Cross sections for electron production by hep neutrinos in the SK and SNO corresponding energy ranges. The ratio of hep to ${ }^{8} \mathrm{~B}$ contributions in each bin is also given, assuming

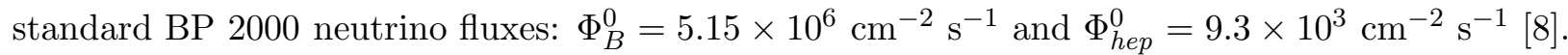
The last column gives the integral difference among the SK and SNO response functions for the reference case of "large hep flux" ( $f_{h e p}=3$ and $\left.f_{B}=1\right)$, as discussed in the text.

\begin{tabular}{|c|c|c|c|c|c|c|c|}
\hline $\begin{array}{c}\text { Range } \\
\quad i\end{array}$ & $\begin{array}{c}\sigma_{h e p}^{e} \\
\left(10^{-44} \mathrm{~cm}^{2}\right)\end{array}$ & $\begin{array}{c}\sigma_{\text {hep }}^{a} \\
\left(10^{-45} \mathrm{~cm}^{2}\right)\end{array}$ & $\begin{array}{c}\sigma_{h e p}^{c} \\
\left(10^{-42} \mathrm{~cm}^{2}\right)\end{array}$ & $\begin{array}{c}\sigma_{\text {hep }}^{e} / \sigma_{B}^{e} \\
\times \Phi_{\text {hep }}^{0} / \Phi_{B}^{0}\end{array}$ & $\begin{array}{c}\sigma_{\text {hep }}^{a} / \sigma_{B}^{a} \\
\times \Phi_{\text {hep }}^{0} / \Phi_{B}^{0}\end{array}$ & $\begin{array}{c}\sigma_{\text {hep }}^{c} / \sigma_{B}^{c} \\
\times \Phi_{\text {hep }}^{0} / \Phi_{B}^{0}\end{array}$ & $\begin{array}{c}\Delta\left(f_{\text {hep }}=3\right) \\
\quad \times 100\end{array}$ \\
\hline 1 & 0.2826 & 0.4476 & 1.2478 & 0.004 & 0.004 & 0.003 & 7.2 \\
\hline 2 & 0.2615 & 0.4107 & 1.3449 & 0.004 & 0.004 & 0.003 & 5.4 \\
\hline 3 & 0.2403 & 0.3745 & 1.4285 & 0.005 & 0.005 & 0.004 & 4.1 \\
\hline 4 & 0.2192 & 0.3391 & 1.5137 & 0.005 & 0.005 & 0.004 & 3.1 \\
\hline 5 & 0.1982 & 0.3048 & 1.5605 & 0.006 & 0.006 & 0.005 & 2.5 \\
\hline 6 & 0.1777 & 0.2718 & 1.6317 & 0.007 & 0.007 & 0.006 & 2.1 \\
\hline 7 & 0.1579 & 0.2402 & 1.6613 & 0.008 & 0.008 & 0.007 & 2.2 \\
\hline 8 & 0.1388 & 0.2103 & 1.6881 & 0.010 & 0.010 & 0.009 & 2.3 \\
\hline 9 & 0.1207 & 0.1821 & 1.7395 & 0.012 & 0.012 & 0.011 & 2.7 \\
\hline 10 & 0.1037 & 0.1560 & 1.6646 & 0.015 & 0.015 & 0.014 & 3.1 \\
\hline 11 & 0.0880 & 0.1319 & 1.6893 & 0.018 & 0.018 & 0.020 & 3.8 \\
\hline 12 & 0.0736 & 0.1100 & 1.5465 & 0.024 & 0.024 & 0.024 & 4.4 \\
\hline 13 & 0.2576 & 0.3823 & 1.2133 & 0.057 & 0.057 & 0.041 & 13.2 \\
\hline
\end{tabular}




\section{SK-SNO corresponding energy ranges}

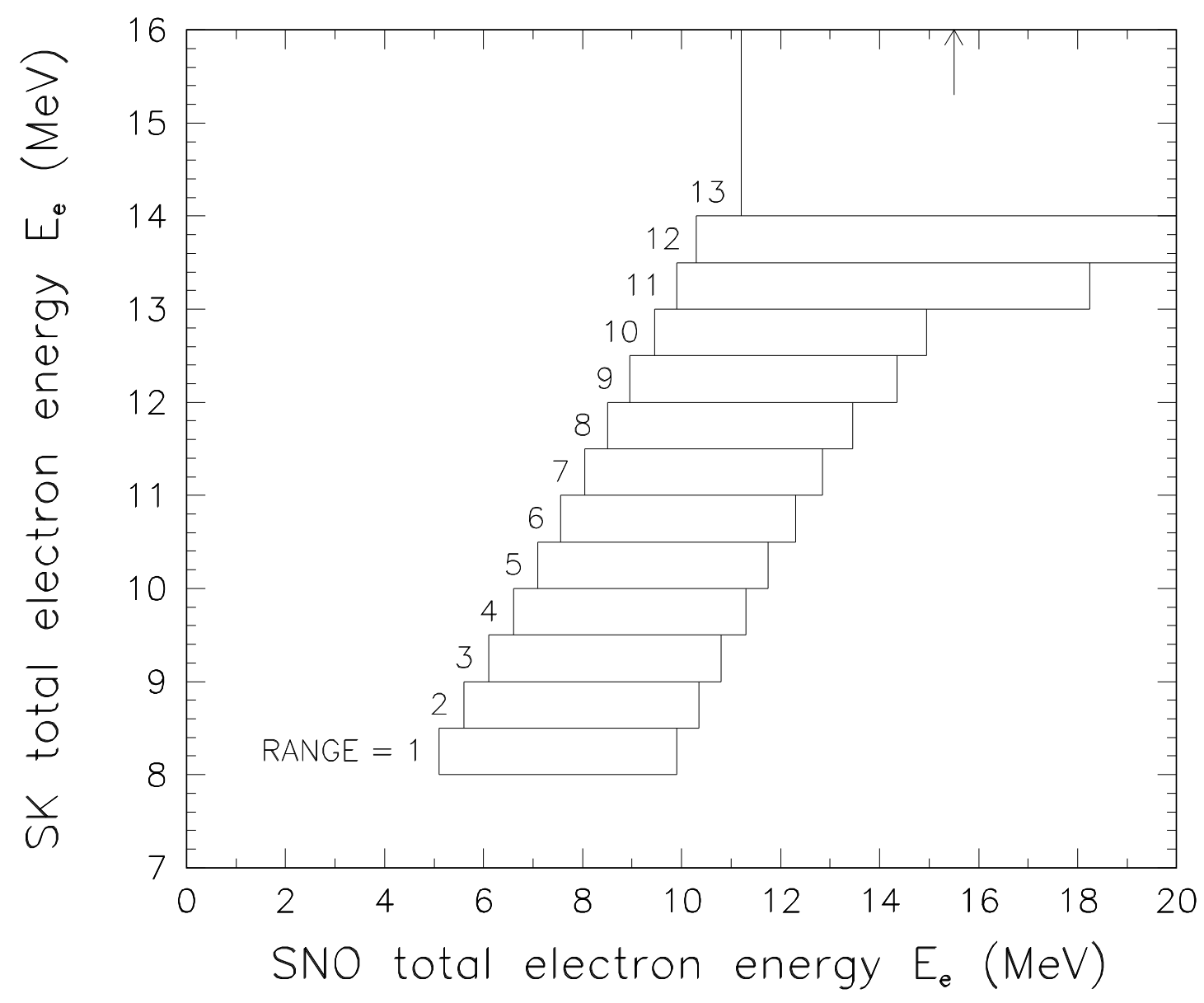

FIG. 1. Correspondence between electron energy ranges in SK and SNO. For each SK energy bin on the $y$-axis, the associated energy range in SNO is given on the $x$-axis. Ranges are labelled by sequential numbers. See also Table I. 


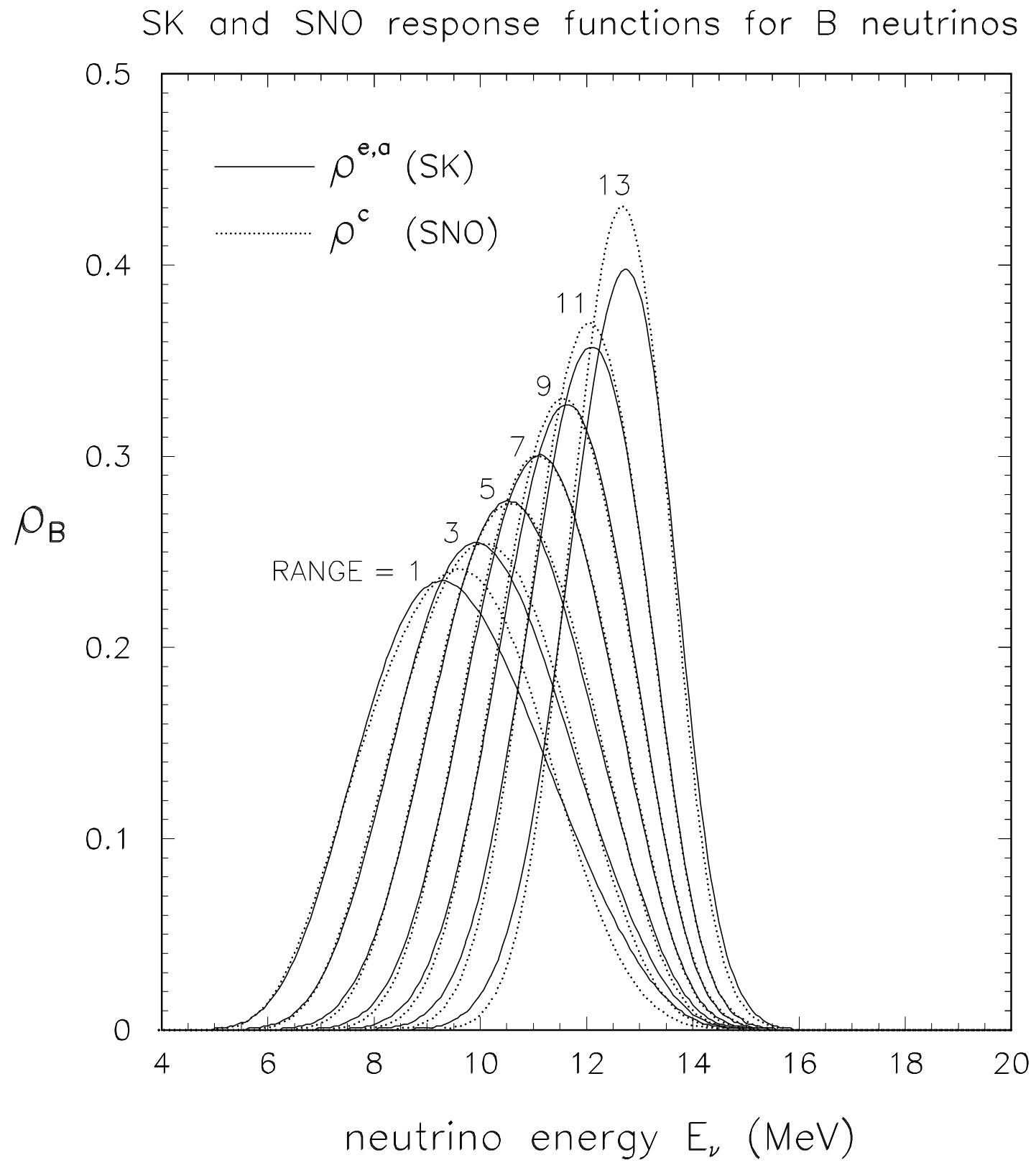

FIG. 2. SK and SNO response functions to ${ }^{8} \mathrm{~B}$ neutrinos in a representative set of energy ranges. The functions are nearly coincident ("equalized") within a few percent. 


\section{SK and SNO electron energy spectra}
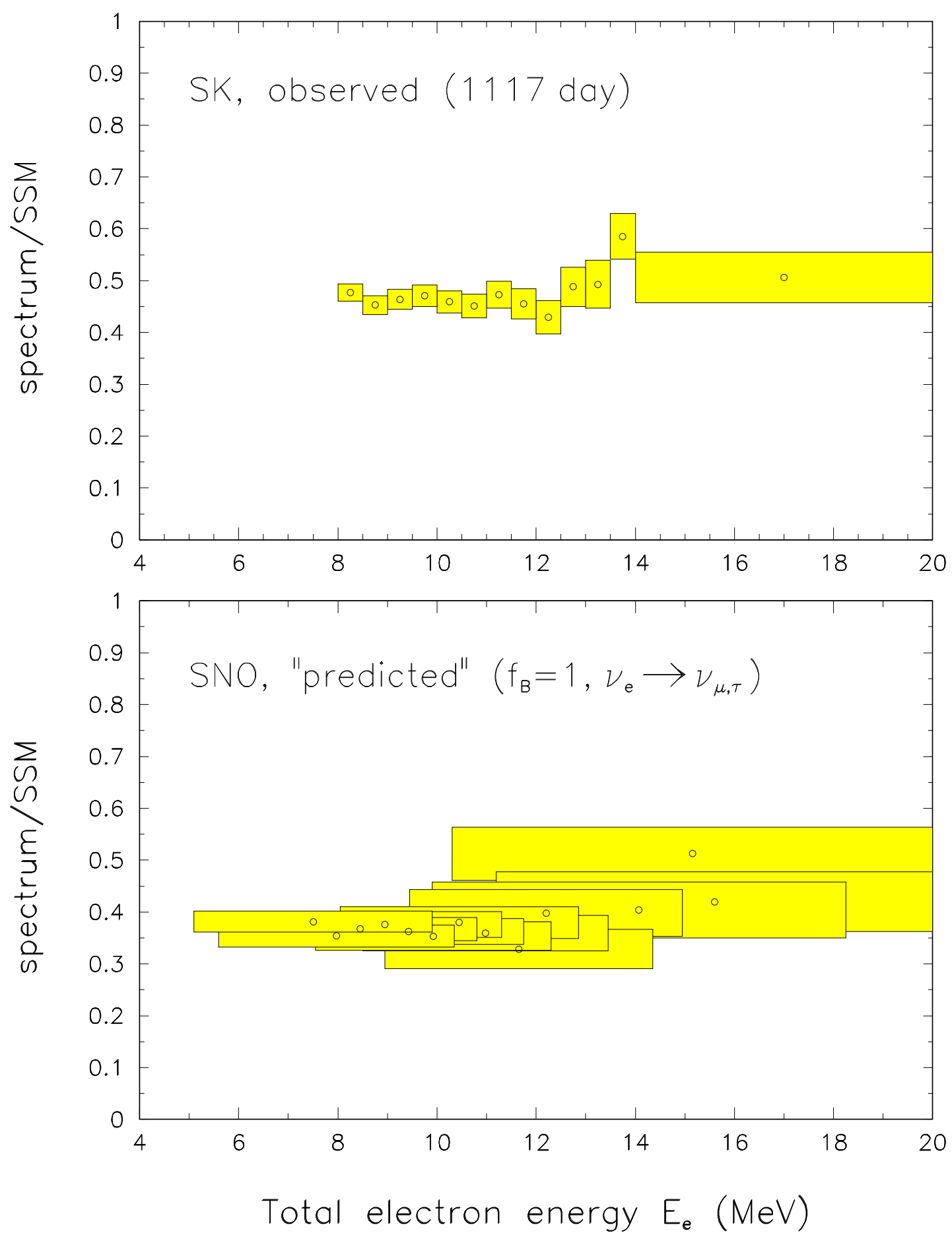

FIG. 3. Upper panel: SK observed energy spectrum (1117 day exposure 110), normalized to the BP 2000 expectations for ${ }^{8} \mathrm{~B}$ neutrinos [8]. The width and height of each grey box represent the bin energy range and the $\pm 1 \sigma$ statistical uncertainty, respectively. Lower panel: SNO "predicted" energy spectrum, as obtained by projecting each SK energy bin rate onto the SNO correlated energy range (and propagating the SK statistical errors). The "prediction" (made for $f_{B}=1$ ) is valid for active oscillations, independently of the functional form of the flavor transition probability. See the text for details. 


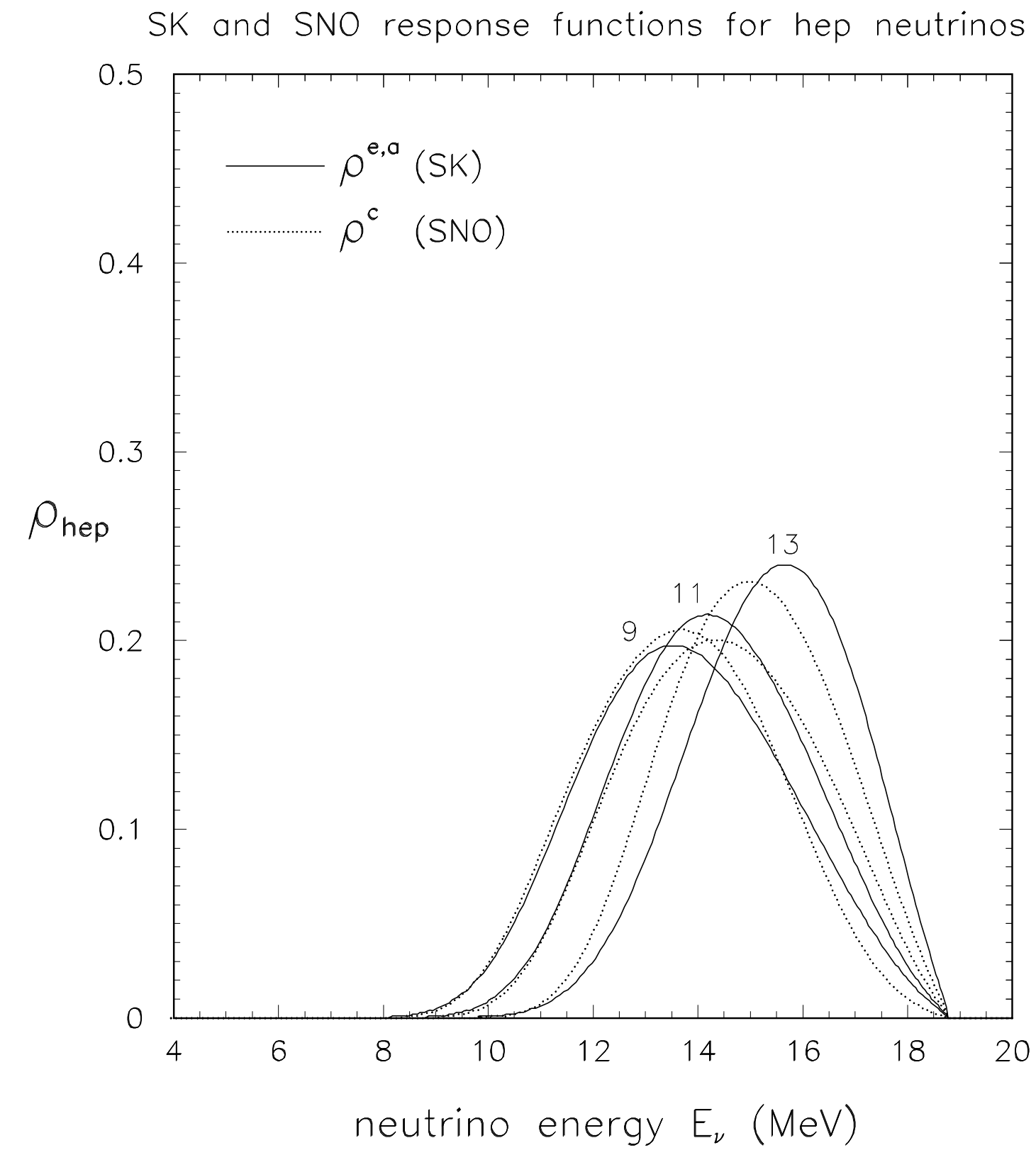

FIG. 4. SK and SNO response functions to hep neutrinos for three representative (high) energy ranges. 


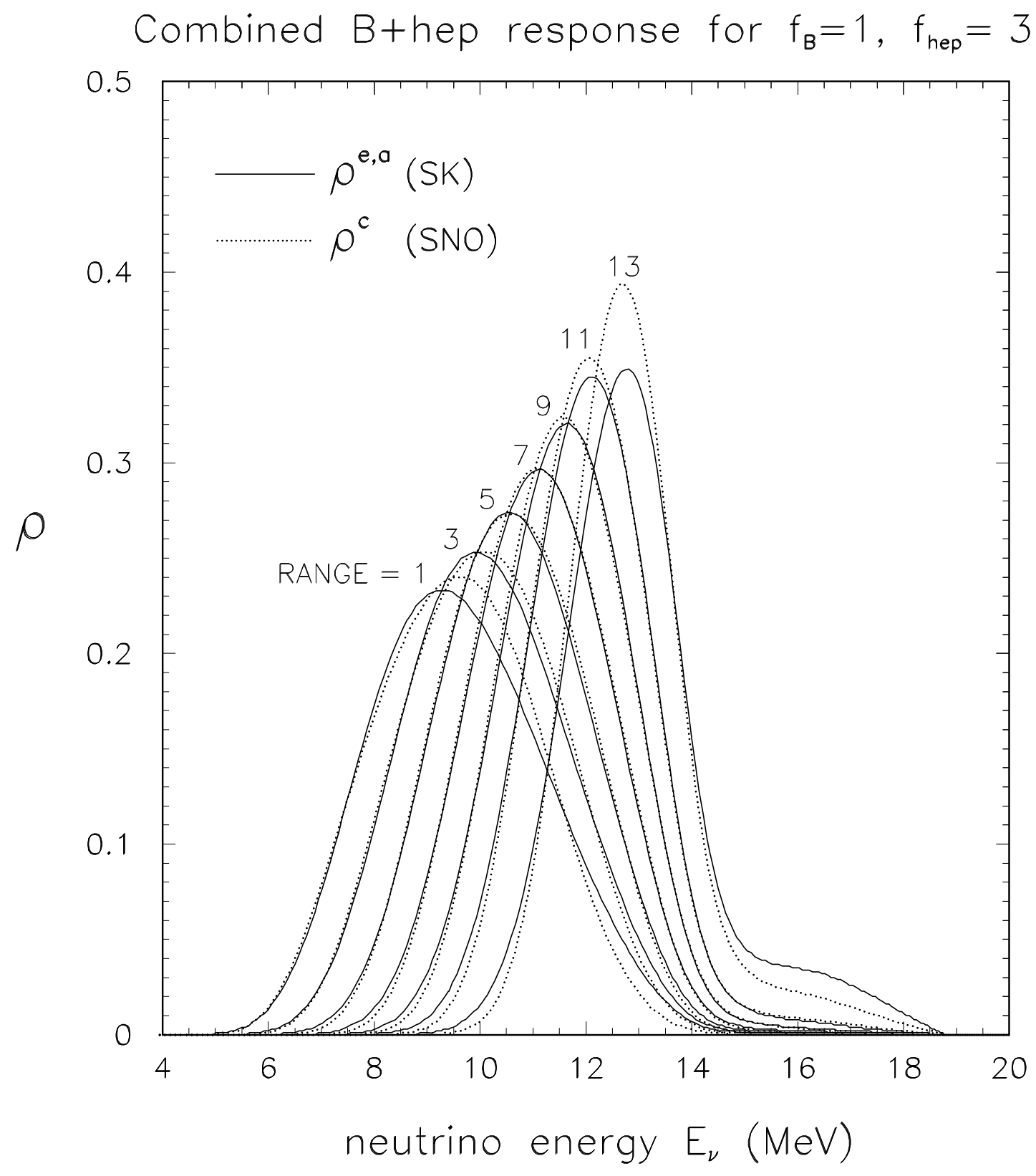

FIG. 5. Combined SK and SNO response functions to ${ }^{8} \mathrm{~B}$ and hep neutrinos, assuming a hep neutrino flux three times larger than the BP 2000 expectations [8]. The functions are approximately coincident (typically within a few percent). A comparison with Fig. 2 shows that the hep contribution modifies the upper tail of the curves for the highest energy ranges, but does not really spoil the approximate equality between the SK and SNO response functions. 


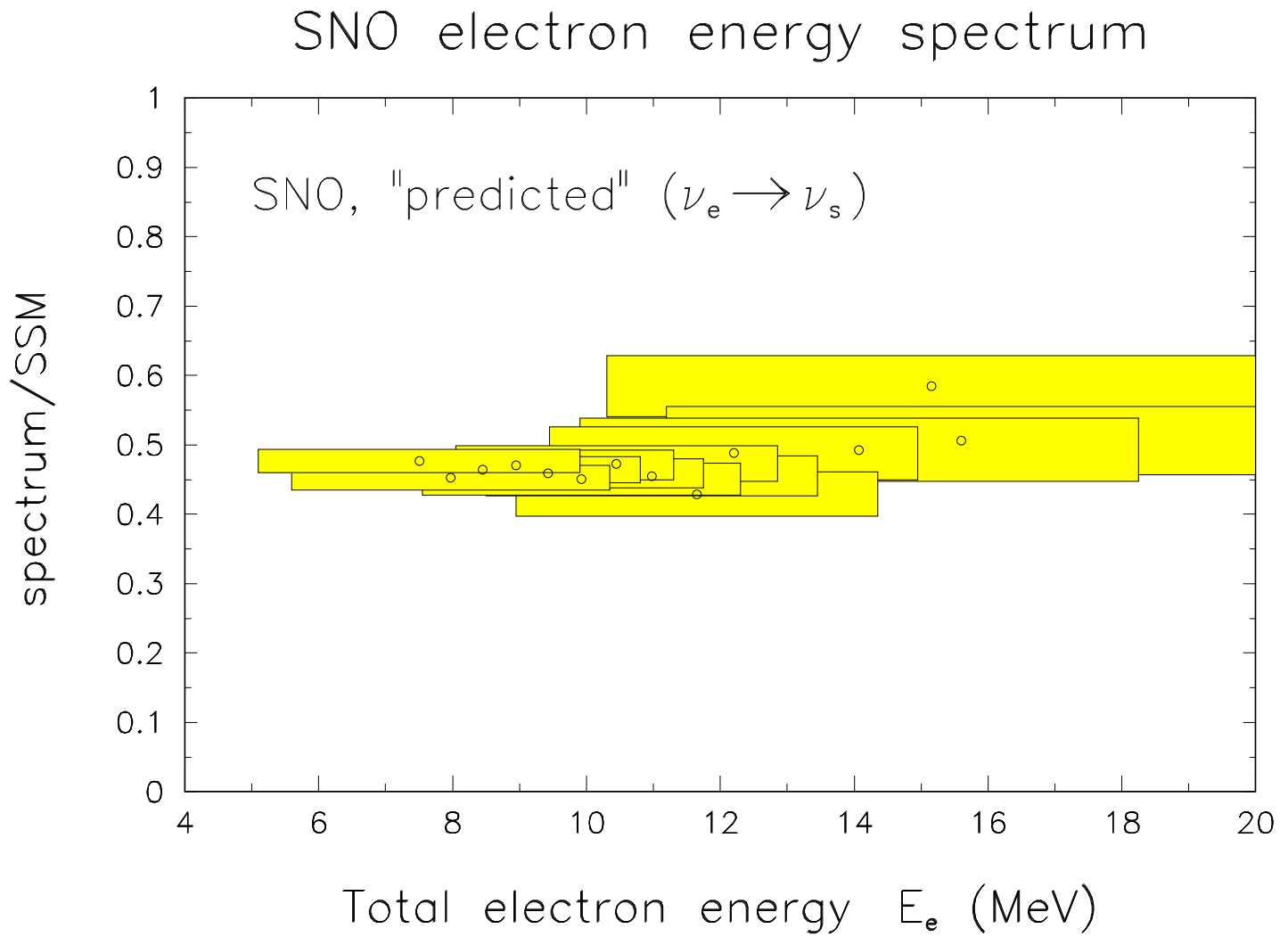

FIG. 6. As in the lower panel in Fig. 3, but for the case of sterile neutrino oscillations. 